Tuberc Respir Dis 2012;73:93-99

Copyright(C)2012. The Korean Academy of Tuberculosis and Respiratory Diseases. All rights reserved

\title{
The Efficacy of Immediate Diet for Reducing Local Adverse Events of Inhaled Corticosteroid: A Pilot Study
}

\author{
Myoung Kyu Lee, M.D., Won Yeon Lee, M.D., Suk Joong Yong, M.D., Kye Chul Shin, M.D., Chong Whan \\ Kim, M.D., Ji-Ho Lee, M.D., Saehyun Jung, M.D., Ye-Ryung Jung, M.D., Hyun Sik Kim, M.D., Tae-Sun Yu, \\ M.D., Sang-Ha Kim, M.D. \\ Department of Internal Medicine, Yonsei University Wonju College of Medicine, Wonju, Korea
}

Background: Local adverse events associated with inhaled corticosteroid use, including dysphonia, pharyngitis and oral candidiasis, can affect adherence for treatment. 'Mouth rinsing method' has been used for reducing local adverse events, but it cannot ensure complete prevention. The goal of this pilot study was to identify whether the 'immediate diet method' can reduce local adverse events in a limited number of patients.

Methods: The study was conducted in a total of 98 patients, who had been prescribed a medium-dose fluticasone propionate for the first time, from January to October in 2010. One training nurse had performed the education on how to use the inhaler, including the mouth rinsing method. And with follow-ups at one month intervals, any patient who experienced such adverse events were educated on the immediate diet method, having a meal within 5 minutes after using an inhaler and they were checked on any incurrence of adverse events with one month intervals for 2 months.

Results: The mean age of patients was 65.9 years old. The local adverse events had incurred from $18.4 \%$ of the study subjects. When performed the follow-up observation in 18 patients with local adverse events after education on the immediate diet method, 14 patients (77.8\%) had shown symptomatic improvements. Three of 4 patients did not show any improvement, in spite of implementing the immediate diet method. The other 1 patient did not practice the immediate diet method properly.

Conclusion: The immediate diet method may be useful in reducing the local adverse events, caused by the use of inhaled corticosteroid.

Key Words: Steroids; Drug Toxicity; Candidiasis, Oral; Pharyngitis; Dysphonia

\section{Introduction}

Glucocorticoids are the most potent and reliable of the available agents among the anti-inflammatory drugs, and have assumed a major role in the management of asthma $^{1}$. However, long-term use of inhaled corticosteroids, especially at higher doses, has been accom-

Address for correspondence: Sang-Ha Kim, M.D. Department of Internal Medicine, Yonsei University Wonju College of Medicine, 162, Ilsan-dong, Wonju 220-701, Korea Phone: 82-33-741-1234, 0926, Fax: 82-33-741-0928

E-mail: sanghakim@yonsei.ac.kr

Received: Feb. 23, 2012

Revised: Apr. 13, 2012

Accepted: Jun. 26, 2012

(a) It is identical to the Creative Commons Attribution Non-Commercial License (http://creativecommons. org/licenses/by-nc/3.0/). panied by concern about systemic complications as well as local adverse events ${ }^{2}$. Systemic complications of high dose inhaled steroids include osteoporosis, adrenocortical suppression, skin thinning, cataract, glaucoma and others $^{3}$. Local adverse events include throat pain or sore throat, dysphonia, oral candidiasis, and others ${ }^{4}$.

Local adverse events of inhaled steroids are reported to be approximately $5 \sim 70 \%{ }^{4}$, and few studies have been reported because most of symptoms are mild compared to systemic complications. But, if appropriate managements of local adverse events are not taken, compliance of patients with inhaled steroids will be decreased and this may influence treatment of diseases. So the efforts that minimize local adverse events are needed. 
The mouth rinsing method is currently implemented to prevent local adverse events by cleansing mouth immediately after taking inhaled corticosteroids. The mouth rinsing method has been practiced based on experience, and few studies have been made regarding the method. Toogood et al. ${ }^{5}$ clinically identified oral candidiasis in $13 \%$ of asthma patients who took high dose inhaled corticosteroids. Moreover, Selroos and Halme $^{6}$ verified that plasma cortisol concentrations were significantly lower $(\mathrm{p}=0.007)$ in patients with rinsing mouth than not rinsing mouth when high dose of budesonide $(1,600 \mu \mathrm{g})$ was inhaled using dry powder inhaler (DPI). But, despite the practice of the mouth rinsing method, local adverse events induced by inhaled corticosteroids still result constraints in the use of medication to patients. For this reason, this study was established a hypothesis that the remained dry powder will be effectively removed when the patients do the method of immediate food intake after using inhaled corticosteroids. And since the administration methods of oral medications have been mostly recognized in relation to meals, the effects of training is thought to be accepted more generally when the use of inhaler devices will be guided as well. Therefore, the authors conducted a pilot study in order to verify the effectiveness of the immediate diet method reducing the occurrence of local adverse reactions.

\section{Materials and Methods}

\section{Study subjects}

This prospective cross-sectional study was comprised a total of 98 patients who visited the respiratory center in a university hospital and were diagnosed with asthma or chronic obstructive pulmonary disease in between January 1st and October 31st in 2010. Consequently, the subjects were prescribed inhaled corticosteroid and long-acting beta-2-agonist complex, Seretide Diskus 250 (fluticasone $250 \mu \mathrm{g} /$ salmeterol $72.5 \mu \mathrm{g}$; GlaxoSmithKline, Seoul, Korea), for the first time. This study was conducted after gaining approval through the deliberations of Institutional Review Board.

\section{Study design}

Subjects was comprised adults aged 19 or above, diagnosed with asthma or chronic obstructive pulmonary disease and prescribed with inhalation of Seretide Diskus 250 twice a day (fluticasone $500 \mu \mathrm{g} /$ day as inhaled steroid) for the first time. On the day of prescription, the subjects were instructed with directions of inhaler including conventional mouth rising method from the nurse who is in charge of training patients. Regarding the instructions for inhaler use, one trained nurse was only responsible for educating patients. The follow-up observation period on local adverse events were identified to be an interval of one month. In case of detection of local adverse events, the immediate diet method was instructed in the use of inhaler instead of the mouth rinsing method. The presence of local adverse reactions was observed over a two-month follow-up period at one month interval.

1) Instructions for the use of inhaler (the mouth rinsing method): The nurse exclusively in charge of training patients performed instructions for use of DPI by applying unified protocol. The directions of unified protocol are as follows:

(1) Hold a diskus with one hand and open the inhaler by pushing the cover with the thumb of the other hand until you hear a 'click' sound.

(2) Hold the device in a way you can see the mouthpiece and make an inhalable state by pushing the lever until you hear a 'click' sound.

(3) After breathing out completely, seal your lips around the mouthpiece and inspire very deep and fast at a consistent speed.

(4) Breathe out after holding your breath for $10 \mathrm{sec}-$ onds or until you feel you cannot breathe in any longer.

(5) Close the inhaler by pushing the cover until you hear a 'click' sound.

(6) Immediately rinse your mouth with water to completely eliminate the remaining dry powder in the mouth right after using inhaler (the mouth rinsing method). 
The protocol comprises a total of six stages. The training was conducted with illustrations and demonstration of the trained nurse who is in charge of instructing patients and approximately 10 minutes were spent per patient.

2) Local adverse events: The presence of local adverse events including sore throat, hoarseness, and oral candidiasis were examined after using inhaler. One month after using inhaler, the occurrence of local adverse events was investigated through medical examination by interviewing patients' subjective symptoms and intra-oral examination. Patients' subjective symptoms were included scratchy or dry throat, throat pain when swallowing, hoarseness, voice change, and others. The oral examination was conducted regarding tongue coating and throat inflammation to examine the occurrence of local adverse events. Sore throat was defined by examining symptoms including scratchy or dry throat, throat pain when swallowing. Dysphonia was determined by observing symptoms including hoarseness, voice change. And oral candidiasis was diagnosed by determining the presence of white coated tongue and throat through subjective oral examination. But fungus culture was not conducted in the coated area of tongue and throat.

3) Instructions for the immediate diet method: The immediate diet method was initiated to patients experiencing local adverse reactions after the using DPI with proper instructions. The instructions for the immediate diet method were identical with the instructions for using DPI until fifth stage. And at the last 6th stage of the DPI protocol, the instruction of "immediately wash your mouth with water" was changed to "immediately have meals" instead. Patients were asked to use the inhaler twice every morning and evening according to general usage and have ordinary meals or meals of at least five or more spoons of rice. Patients were directed to use the inhaler right before having meals and to have a meal within five minutes after using the DPI for better understanding of the term, "the immediate diet method." Symptom improvement of patients guided with the immediate diet method was checked for the two-month follow-up period at one month interval. Other oral medications or gargling drugs were not prescribed to patients instructed the immediate diet method because those medications could be beneficial to local adverse reactions.

4) Statistical analysis: SPSS version 15.0 (SPSS Inc., Chicago, IL, USA) was used for statistical analysis and t-test were performed to compare the results of pulmonary function test and the age difference between two groups with local adverse events and without local adverse events after using inhalation device. Chi-square analysis was conducted to compare the differences between genders and prevalence rate of causal diseases. Continuous variables are shown as mean \pm standard deviation. A p-value less than 0.05 was regarded as statistically significant.

\section{Results}

\section{Local adverse events and clinical manifestations after using the inhaled corticosteroids}

A total of 98 subjects were prescribed with the same diskus containing inhaled corticosteroids and patients were 50 males and 48 females. The average age of pa-

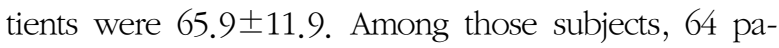
tients were diagnosed with asthma and 34 patients were diagnosed with chronic obstructive pulmonary disease. Among 98 patients, local adverse events were detected in 18 patients (18.4\%), comprising 7 males and 11 females. Local adverse events were observed in 13 patients (20.3\%) diagnosed with asthma and 5 patients (13.7\%) diagnosed with chronic obstructive pulmonary disease but no statistical significance was found $(p=$ 0.070). In terms of local adverse events, oral candidiasis, sore throat, and dysphonia patients were 7 (7.1\%), 6 (6.1\%) and 5 (5.1\%), respectively (Figure 1). The average age of patients experiencing local adverse events was $70.3 \pm 11.4$, relatively higher than 64.9 \pm 11.8 , the average age of patients with no local adverse events. There was no statistical significance $(p=0.083)$. According to pulmonary function test results before and after the use of inhaled bronchodilators implemented in 
MK Lee et al: Immediate diet for local adverse events of inhaled corticosteroid

two groups, no statistical significance was found in indicators including forced expiratory volume in one second $\left(\mathrm{FEV}_{1}\right)$, forced vital capacity (FVC), $\mathrm{FEV}_{1} / \mathrm{FVC}$, and others (Table 1).

2. Results of implementation of the immediate diet method in patients experiencing local adverse events

A total of 18 patients experiencing local adverse

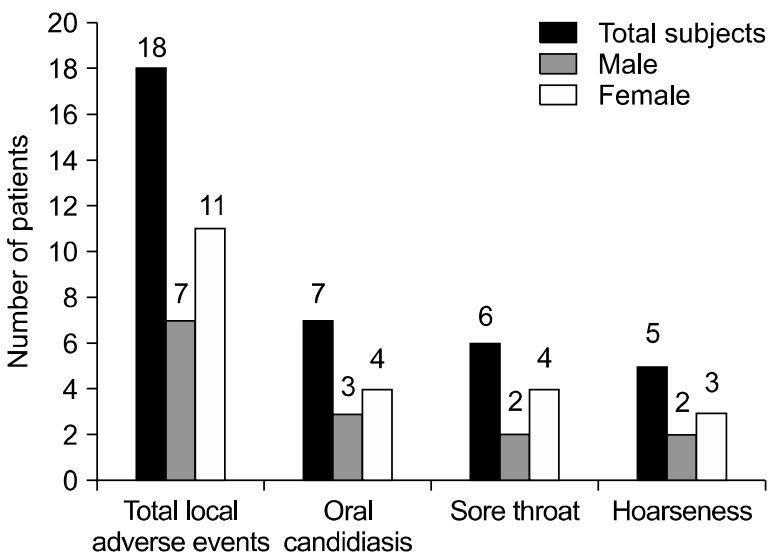

Figure 1. The prevalence of local adverse events of inhaled corticosteroids. When checked on any incurrence of adverse events at 98 patients, who had been prescribed a medium-dose fluticasone propionate with one month interval for 2 months, a total 18 patients experienced local adverse events (7 men, 11 women). Of those 18 patients, 7 patients developed oral candidiasis ( 3 men, 4 women), 6 patients incurred sore throat (2 men, 4 women), and 5 patients developed hoarseness ( 2 men, 3 women). events were examined for the two-month follow-up period at one month interval after they were instructed with the immediate diet method. Symptoms of $14 \mathrm{pa}-$ tients (77.8\%) were alleviated (Figure 2). In the first month of follow-up period, 13 patients' symptoms were relieved. Symptoms were improved in 6 patients with sore throat, 5 patients with oral candidiasis, and 2 pa-

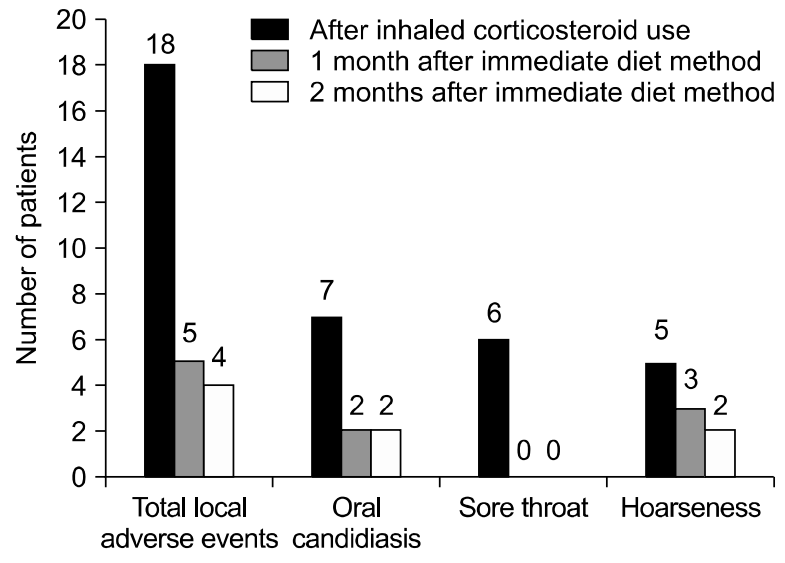

Figure 2. Changes of the prevalence of local adverse events of inhaled corticosteroid after education of the immediate diet. When performed the follow-up observation in 18 patients with local adverse events after education on the immediate diet, 13 patients had shown symptomatic improvements 1 month later, and total of 14 patients had shown symptomatic improvements 2 months later. Five of 7 patients with oral candidiasis had improved 2 months later; all of 6 patients with sore throat had improved after 2 months, and 3 of 5 patients with hoarseness had improved 2 months later with a follow-up observation.

Table 1. Clinical characteristics of studied subjects according to the presence of local adverse events after use of inhaled corticosteroid

\begin{tabular}{lcrc}
\hline \multicolumn{1}{c}{ Variables } & With local adverse events $(n=18)$ & Without local adverse events $(n=80)$ & $p$-value \\
\hline Age, yr & $70.3 \pm 11.4$ & $64.9 \pm 11.8$ & 0.083 \\
Sex, male, \% & $7(38.9)$ & $43(53.8)$ & 0.082 \\
Asthma, \% & $13(20.3)$ & $51(79.7)$ & 0.196 \\
Diabetes mellitus, \% & $2(40.0)$ & $3(60.0)$ & 0.184 \\
Pre-FEV,$\%$ predicted & $49.6 \pm 19.1$ & $59.1 \pm 26.3$ & 0.149 \\
Pre-FEV $/$ FVC, \% & $63.3 \pm 16.4$ & $57.6 \pm 15.0$ & 0.157 \\
Post-FEV,$\%$ predicted & $55.3 \pm 17.9$ & $63.7 \pm 25.5$ & 0.227 \\
Post-FEV $/$ FVC, \% & $62.8 \pm 13.7$ & $59.5 \pm 14.7$ & 0.417 \\
\hline
\end{tabular}

$\mathrm{FEV}_{1}$ : forced expiratory volume in one second; FVC: forced vital capacity. 
tients with dysphonia. And in the second month of follow-up period, symptom of one patient experiencing dysphonia was alleviated. Symptoms of four patients (22.2\%) with no improvement persisted, and there were two patients each in oral candidiasis and dysphonia (Figure 2). Although the immediate diet method was conducted in three patients experiencing adverse events among four patients with no improvement, symptoms were not alleviated. For this reason, prescribed medications had been changed or ceased. There was one patient who did not properly conduct the immediate diet method.

\section{Discussion}

Among 98 subjects, 18 of them experienced local adverse events after taking inhaled corticosteroids. When the immediate diet method was instructed to those 18 patients, symptoms of 14 patients (77.8\%) were relieved. Although mechanisms of improvement are unknown, the immediate diet method is thought to facilitate the elimination of dry power remaining in the mouth. And reducing the amount of steroid powder remaining in the mouth is known as a preventive measure for local adverse events. Yokoyama et al. ${ }^{7}$ identified that the amount of steroid powder was reduced more than $90 \%$ when mouth rinsing was performed up to five times than when it was performed only once in patients who inhaled fluticasone. The results were demonstrated that local adverse events such as oral candidiasis are prevented when mouth rinsing is performed repeatedly to remove the remaining powder inducing adverse reactions. Similarly in our study, the reason for the reduced occurrence of local adverse events by using the immediate diet method was assumed that was due to removal of steroid powder in the mouth. When the improvement of each local adverse reaction was examined, six patients with sore throat were all improved after being trained with the immediate diet method. In case of patients with oral candidiasis, five out of seven got better after being trained with the immediate diet method. Moreover, three out of five patients with dys- phonia showed improvement. The immediate diet method was suggested to be more effective especially in sore throat. However, further comparative studies are essential with larger study population to verify this prediction.

The incidence of local adverse events upon the use of inhaled corticosteroids vary from 5 70\% depending on researchers ${ }^{4,8,9}$. The occurrence of local adverse events differs depending on the ages of patients and types, container, and dose of inhalation devices. Dubus et al. ${ }^{8}$ presented that $60 \%$ of pediatric patients reported local adverse events after taking inhaled corticosteroids. Pauwels et al. ${ }^{10}$ reported that the incidence of cough, dysphonia, throat pain and other events were significantly increased when inhaled corticosteroids were taken using metered dose inhaler (MDI) instead of DPI $(\mathrm{p}=0.0001)$. According to meta-analysis in connection with this result, the risk of incidence was five times higher than placebo group in case of oral candidiasis when steroids were inhaled using the MDI. The risk of incidence was three times higher when DPI was used ${ }^{11}$. Our study was comprised relatively older patients with the average age of 66 . The prevalence rate of local adverse events was $18.4 \%$ when the patients took the drugs containing inhaled corticosteroids using DPI. Besides from oral candidiasis, dysphonia, and sore throat, other local adverse events include dermatitis around lips, cough, dry mouth, tongue hypertrophy, and others ${ }^{4}$. In addition, there are some patients reporting toothache and stomatitis ${ }^{12}$. However, in this study, there was no patient reporting symptoms other than oral candidiasis, sore throat, and dysphonia.

When mechanisms of those local adverse events were examined, immune functions of neutrophils, macrophages, and T-lymphocytes existing within the oral cavity were inhibited due to accumulation of inhaled corticosteroids in the mouth. This eventually leads to locally weakened immune system and increased glucose concentration in salivary glands, resulting rapid growth of Candida species ${ }^{13}$. Dysphonia is known to be resulted from deformation of vocal folds due to myopathy induced by accumulation of steroids in the muscles of vo- 
cal cord ${ }^{14}$. Sore throat has been reported to be caused by secondary infection in the throat due to over-stimulated mucous membranes and weakened immune system $^{15}$. Therefore, when the patients with local adverse events perform the proper implementation of mouth rinsing or the immediate diet method, the amount of accumulated steroids within the oral cavity needs to be reduced. Besides, weakened local immune system in the throat will be enhanced. Moreover, secondary growth of Candida species and throat infection will be prevented by reducing glucose concentration in salivary glands, leading to decreased adverse events including oral candidiasis, sore throat, and others. In addition, dysphonia induced by deformation of vocal cords is thought to be reduced by lowering accumulation of corticosteroids within the muscles.

Currently, mouth fresheners or gargles containing antifungal agents are prescribed for treatment in case of incidence of local adverse events such as oral candidiasis $^{16}$. When inhaled corticosteroids are used, patients are mostly instructed to practice mouth rinsing by immediately washing mouth with water to prevent the occurrence of local adverse events. In case of oral candidiasis, using MDI with spacer, or decrease in the frequency of inhaled corticosteroids are encouraged to reduce the amount of steroid powder remaining in the mouth. However, no clarification has been made on if up to what extent local adverse events could be reduced or prevented ${ }^{6}$.

One of the limitations in this study is that the research was performed without control group even though it was a pilot study. To compare preventive effectiveness on local adverse events arising from inhaled corticosteroids, further studies are crucial with control groups in each of the mouth rinsing method currently applied based on previous experience and newly proposed the immediate diet method. Confirming possibilities of the preventive measures is essential for the progress of further studies. Second, no assessment or analysis has been made regarding on the difference varying depending on skillfulness of individual patient in the usage of inhalation device. However, this study was conducted on patients using the same container (diskus, DPI), and identical inhaled corticosteroids (fluticasone, $500 \mu \mathrm{g} /$ day) in the same dose. To minimize confusing factors, instructions with standardized methods were given by the trained nurse who was exclusively in charge of training patients regarding the use of inhalation device. Furthermore, according to a comparative study of Brand et al. ${ }^{17}$ recently conducted on the MDI, accumulation of steroids within the oral cavity before and after the appropriate training of inhaler technique did not shown significant difference from 56\% to 55\%. According to a study of Lee and Yang ${ }^{18}$, although knowledge and accuracy in the usage of inhalation device was increased with proper instructions conducted on patients, satisfaction on the use of inhaler was not increased and peak expiratory flow and instability in asthma control was not shown improvement either. Consequently, continuous trainings and elapsed time seemed to have almost no influence on the reduction of adverse reactions and the enhancement of pulmonary function. Additional studies are thought to be essential to investigate on the effectiveness of inhalation device training on reduction of steroids in the mouth and local adverse events. To progress further studies, analysis on the incidence of local adverse events needs to be performed collectively according to skillfulness assessed after training patients.

In summary, the immediate diet method is a method that having meals immediately after using inhaled corticosteroids to minimize local adverse reactions. This method is expected to be beneficial for reducing local adverse events of inhaled devices. So the results of this study will be used as basic data in upcoming comparative studies verifying effectiveness of the mouth rinsing method and the immediate diet method for reducing local adverse events.

\section{References}

1. Szefler SJ. Glucocorticoid therapy for asthma: clinical pharmacology. J Allergy Clin Immunol 1991;88:147-65.

2. Hanania NA, Chapman KR, Kesten S. Adverse effects of inhaled corticosteroids. Am J Med 1995;98:196-208.

3. Maxwell DL. Adverse effects of inhaled corticosteroids. 
Biomed Pharmacother 1990;44:421-7.

4. Roland NJ, Bhalla RK, Earis J. The local side effects of inhaled corticosteroids: current understanding and review of the literature. Chest 2004;126:213-9.

5. Toogood JH, Jennings B, Greenway RW, Chuang L. Candidiasis and dysphonia complicating beclomethasone treatment of asthma. J Allergy Clin Immunol 1980;65:145-53.

6. Selroos O, Halme M. Effect of a volumatic spacer and mouth rinsing on systemic absorption of inhaled corticosteroids from a metered dose inhaler and dry powder inhaler. Thorax 1991;46:891-4.

7. Yokoyama H, Yamada Y, Yamamura Y, Nakamura H, Iga $\mathrm{T}$. Effect of mouth wash on the removing fluticasone propionate delivered by dry powder inhaler in mouth. Yakugaku Zasshi 2001;121:233-7.

8. Dubus JC, Marguet C, Deschildre A, Mely L, Le Roux $\mathrm{P}$, Brouard J, et al. Local side-effects of inhaled corticosteroids in asthmatic children: influence of drug, dose, age, and device. Allergy 2001;56:944-8.

9. Toogood JH. Complications of topical steroid therapy for asthma. Am Rev Respir Dis 1990;141(2 Pt 2):S89-96.

10. Pauwels RA, Hargreave FE, Camus P, Bukoski M, Ståhl E. A 1-year comparison of turbuhaler vs pressurized metered-dose inhaler in asthmatic patients. Chest 1996;110:53-7.

11. Rachelefsky GS, Liao Y, Faruqi R. Impact of inhaled corticosteroid-induced oropharyngeal adverse events: results from a meta-analysis. Ann Allergy Asthma Immunol 2007;98:225-38.

12. Richter K, Kanniess F, Biberger C, Nave R, Magnussen $\mathrm{H}$. Comparison of the oropharyngeal deposition of inhaled ciclesonide and fluticasone propionate in patients with asthma. J Clin Pharmacol 2005;45:146-52.

13. Knight L, Fletcher J. Growth of Candida albicans in saliva: stimulation by glucose associated with antibiotics, corticosteroids, and diabetes mellitus. J Infect Dis 1971;123:371-7.

14. Williams AJ, Baghat MS, Stableforth DE, Cayton RM, Shenoi PM, Skinner C. Dysphonia caused by inhaled steroids: recognition of a characteristic laryngeal abnormality. Thorax 1983;38:813-21.

15. Buhl R. Local oropharyngeal side effects of inhaled corticosteroids in patients with asthma. Allergy 2006;61: 518-26.

16. Hood S, Evans J, Bond J, Wilkins E, Denning D. The treatment of oropharyngeal candidiasis in HIV-infected patients with oral amphotericin B suspension. AIDS Patient Care STDS 1998;12:625-7.

17. Brand P, Hederer B, Austen G, Dewberry H, Meyer T. Higher lung deposition with Respimat Soft Mist inhaler than HFA-MDI in COPD patients with poor technique. Int J Chron Obstruct Pulmon Dis 2008;3:763-70.

18. Lee JK, Yang YH. Evaluation of an education program for patients with asthma who use inhalers. J Korean Acad Nurs 2010;40:202-12. 